\title{
A fluid description for Landau damping of dispersive MHD waves
}

\author{
T. Passot and P. L. Sulem \\ CNRS, Observatoire de la Côte d'Azur, B.P. 4229, 06304 Nice Cedex 4, France \\ Received: 30 June 2003 - Revised: 2 December 2003 - Accepted: 5 January 2004 - Published: 14 April 2004 \\ Part of Special Issue "International Workshops on Nonlinear Waves and Chaos in Space Plasmas"
}

\begin{abstract}
The dynamics of long oblique MHD waves in a collisionless plasma permeated by a uniform magnetic field is addressed using a Landau-fluid model that includes Hall effect and electron-pressure gradient in a generalized Ohm's law and retains ion finite Larmor radius (FLR) corrections to the gyrotropic pressure (Phys. Plasmas 10, 3906, 2003). This one-fluid model, built to reproduce the weakly nonlinear dynamics of long dispersive Alfvén waves propagating along an ambient field, is shown to correctly capture the Landau damping of oblique magnetosonic waves predicted by a kinetic theory based on the Vlasov-Maxwell system. For oblique and kinetic Alfvén waves (for which second order FLR corrections are to be retained), the linear character of waves with small but finite amplitudes is established, and the dispersion relation reproduced in the regime of adiabatic protons and isothermal electrons, associated with the condition $m_{e} / m_{p} \ll \beta \ll T_{e} / T_{p}$, where $\beta$ is the squared ratio of the ion-acoustic to the Alfvén speeds. It is shown that in more general regimes, the heat fluxes are, to leading order, not gyrotropic and dependent on the Hall effect to leading order.
\end{abstract}

\section{Introduction}

Magnetohydrodynamic (MHD) wave turbulence is ubiquitous in space plasmas, from the solar wind and planet magnetospheres to the Interstellar Medium (ISM). In the warm and diffuse ISM for example, weak MHD wave turbulence is believed to be responsible for the observed interstellar scintillation through the density fluctuation they produce (Spangler, 1991). A remarkable Kolmogorov spectrum for the density fluctuations is measured over more than twelve decades, down to scales of the order of $100 \mathrm{~km}$, i.e. the typical ion inertial length (Armstrong et al., 1995). The properties of this turbulence are still debated. Questions concern the origin of this extended inertial range, the nature of the ISM phase from where fluctuations originate (possibly also HII regions)

Correspondence to: T. Passot

(passot@obs-nice.fr) and the physical nature of the dissipation processes (Minter and Spangler, 1995; Lithwick and Goldreich, 2001). These aspects are usually addressed separately, the large-scale dynamics being often described in terms of usual ideal compressible MHD, while the small-scale (collisionless) dissipative effects are evaluated using a kinetic approach, assuming a given turbulence energy spectrum. It would thus be desirable to have a fluid description incorporating the dominant kinetic effects such as wave-particle resonance and FLR corrections. Such a model would provide a self-consistent description of the turbulent cascade and allow a realistic estimate of its termination scales. It would in particular contribute to a detailed understanding of collisionless dissipation at the origin of heating, and its balance with cooling processes. Among the various collisionless dissipation mechanisms, Landau damping of magnetosonic waves has recently been shown to provide a dominant contribution (Lerche and Schlickeiser, 2001a). Proper treatment of Landau damping in ISM turbulence is also essential for the determination of the full fast mode spectrum and thus of the properties of the cosmic ray scattering (Lerche and Schlickeiser, 2001b).

Similar problems are encountered in the solar wind where magnetic fluctuations show an abrupt transition from a $-5 / 3$ power law spectrum at large scale to a steeper slope at the scales where proton inertia becomes non-negligible ( $\mathrm{Li}$ et al., 2001). In the magnetosheath, a realistic description of the turbulence reaching the magnetopause could improve the understanding of the high level of magnetic field fluctuations that is believed to be at the origin of micro-reconnection and particle penetration in the magnetosphere (Rezeau and Belmont, 2001).

In order to capture such phenomena, one needs a description going beyond the usual MHD or even the double adiabatic approximation (Chew etal., 1956) that retains plasma anisotropy but neglects heat fluxes. A significant contribution in the development of such a tool is provided by the Landau-fluid models of Snyder et al. (1997). A simplified version of this approach was used by Quataert et al. (2002) to describe the magneto-rotational instability in collisionless 
accretion disks. Such models are however to be generalized when the fluctuations extend to scales comparable to the ion inertial length, a regime where finite Larmor radius (FLR) corrections to the pressure tensor, together with Hall effect and electron pressure tensor gradient in a generalized Ohm's law have to be retained. Such an extension developed by Passot and Sulem (2003b), is described in Sect. 2. In Sect. 3, this model is shown to accurately reproduce the Landau damping of long magnetosonic waves propagating in an oblique direction, as predicted by the Vlasov-Maxwell kinetic theory performed in Appendix A. Section 4 briefly summarizes the case of parallel Alfvén waves already discussed by Passot and Sulem (2003b) for which the Landau damping does not arise as a linear dissipation but enters the derivative nonlinear Schrödinger equation governing the long-wavelength dynamics (Rogister, 1971; Mjølhus and Wyller, 1988). Sections 5 and 6 deal with obliquely propagating Alfvén waves and with the limiting case of kinetic Alfvén waves respectively. Second order FLR corrections that are relevant in these regimes, are derived in Appendix B. The linear character of the dynamics in the case of waves of small but finite amplitudes is established, and the computed dispersion and damping are shown to reproduce classical results in the regime $\frac{m_{e}}{m_{p}} \ll \beta \ll \frac{T_{e}}{T_{p}}$ where $\beta$ is the squared ratio of the ion-acoustic to the Alfvén speeds. Possible extension of the model to more general conditions are discussed in the Conclusion.

\section{Generalized MHD with Landau damping}

As already mentioned, a fluid description going beyond the usual MHD and including a realistic dissipation mechanism is believed to provide a very efficient tool for modeling largescale turbulence in a collisionless plasma permeated by a strong ambient magnetic field.

Starting with the Vlasov-Maxwell equations, one easily derives a hierarchy of equations for the successive moments of the distribution functions of the various particle species. When reduced to a one-fluid description, the time evolution of the density and velocity of the plasma obeys

$\partial_{t} \rho+\nabla \cdot(\boldsymbol{u} \rho)=0$ $\rho\left(\partial_{t} \boldsymbol{u}+\boldsymbol{u} \cdot \nabla \boldsymbol{u}\right)+\nabla \cdot \mathbf{P}+\frac{1}{4 \pi} \boldsymbol{b} \times(\nabla \times \boldsymbol{b})=0$,

where the pressure tensor $\mathbf{P}$ is defined relatively to barycentric velocities. In a weakly nonlinear regime (strong ambient field), the pressure tensor can be approximated by the sum of the individual pressures of the various species, even at scales comparable with the ion Larmor radius. In an expansion in terms of the ratio of a typical hydrodynamic scale to the ion Larmor radius, the pressure tensor $\mathbf{P}=\mathbf{P}^{G}+\Pi$ is dominated by the gyrotropic contribution

$\mathbf{P}^{G}=\sum_{r} \mathbf{P}_{r}^{G}=\sum_{r} p_{\perp r}(\mathbf{I}-\widehat{\boldsymbol{b}} \otimes \widehat{\boldsymbol{b}})+p_{\| r} \widehat{\boldsymbol{b}} \otimes \widehat{\boldsymbol{b}}$,

where the parallel and perpendicular components $p_{\| r}$ and $p_{\perp r}$ for the individual species are governed by

$$
\begin{aligned}
\partial_{t} p_{\| r}+\nabla \cdot\left(\boldsymbol{u} p_{\| r}\right) & +\nabla \cdot\left(\widehat{\boldsymbol{b}} q_{\| r}\right) \\
+ & 2 p_{\| r} \widehat{\boldsymbol{b}} \cdot \nabla \boldsymbol{u} \cdot \widehat{\boldsymbol{b}}-2 q_{\perp r} \nabla \cdot \widehat{\boldsymbol{b}}=0, \\
\partial_{t} p_{\perp r}+\nabla \cdot\left(\boldsymbol{u} p_{\perp r}\right) & +\nabla \cdot\left(\widehat{\boldsymbol{b}} q_{\perp r}\right)+p_{\perp r} \nabla \cdot \boldsymbol{u} \\
& -p_{\perp r} \widehat{\boldsymbol{b}} \cdot \nabla \boldsymbol{u} \cdot \widehat{\boldsymbol{b}}+q_{\perp r} \nabla \cdot \widehat{\boldsymbol{b}}=0,
\end{aligned}
$$

with $\widehat{\boldsymbol{b}}$ denoting the unit vector along the local magnetic field. The heat flux tensors are furthermore supposed to be dominated by the gyrotropic components $q_{\| r}$ and $q_{\perp r}$, an assumption whose validity will be discussed in Sect. 4.

The finite Larmor radius corrections to the pressure tensor are described by the tensor $\Pi$ that, when electron inertia is negligible, reduces to the proton contribution $\Pi_{p}$. The latter is to be computed perturbatively, the required accuracy depending on the considered regime. Details are given in Appendix B.

With a generalized Ohm's law that includes the Hall effect and the contribution of the electron pressure gradient, the induction equation reads

$$
\begin{aligned}
& \partial_{t} \boldsymbol{b}-\boldsymbol{\nabla} \times(\boldsymbol{u} \times \boldsymbol{b})= \\
&-\frac{m_{p} c}{q_{p}} \nabla \times\left[\frac{1}{4 \pi \rho}(\nabla \times \boldsymbol{b}) \times \boldsymbol{b}-\frac{1}{\rho} \nabla \cdot \mathbf{P}_{e}^{G}\right] .
\end{aligned}
$$

To close this system, one needs to specify the evolution of the heat fluxes. In regimes where these quantities are not sensitive to the Hall effect, Passot and Sulem (2003b) suggested

$$
\begin{aligned}
& \left(\frac{d}{d t}+\frac{v_{t h, r}}{\sqrt{\frac{8}{\pi}}\left(1-\frac{3 \pi}{8}\right)} \mathcal{H} \nabla_{\|}\right) \frac{q_{\| r}}{v_{t h, r} p_{\| r}^{(0)}}=\frac{1}{1-\frac{3 \pi}{8}} v_{t h, r} \nabla_{\|} \frac{T_{\| r}}{T_{\| r}^{(0)}} \\
& \left(\frac{d}{d t}-\sqrt{\frac{\pi}{2}} v_{t h, r} \mathcal{H} \nabla_{\|}\right) \frac{q_{\perp r}}{v_{t h, r} p_{\perp r}^{(0)}}=-v_{t h, r} \nabla_{\|}\left(\frac{T_{\perp r}}{T_{\perp r}^{(0)}}+\left(\frac{T_{\perp r}^{(0)}}{T_{\| r}^{(0)}}-1\right) \frac{|b|}{B_{0}}\right),
\end{aligned}
$$

where $\nabla_{\|}$denotes the derivative and $\mathcal{H}$ the Hilbert transform along the ambient field, and where the temperatures are given by $p_{\| r}=n T_{\| r}$ and $p_{\perp r}=n T_{\perp r}$. The coefficients $v_{t h, r}$ refers to the (equilibrium) thermal velocities of the $r$ species and the superscript (0) to the equilibrium state. This closure can essentially be viewed as a linear version of Eqs. (32-35) of Snyder et al. (1997) and was shown to accurately describe the parallel propagation of weakly nonlinear dispersive Alfvén waves in the long-wavelength limit. Indeed, as discussed in Sect. 4, reductive perturbative expansions performed on the 
above system and on the primitive Vlasov-Maxwell equations lead to asymptotic equations that identify up to the replacement of the plasma response function by suitable Padé approximants.

Note that, consistently with the weakly nonlinear assumption underlying the derivation of the heat flux equations, the Landau damping is evaluated by integration along the ambient field lines, which makes its numerical evaluation easy when using a Fourier spectral code. Convective derivatives are nevertheless retained in Eqs. (7-8) in order to preserve Galilean invariance.

\section{Oblique magnetosonic waves}

We here demonstrate that the model described in Sect. 2 correctly reproduces the dissipation rate of oblique magnetosonic waves predicted by kinetic theory in the longwave limit. Assuming a propagation in a direction making an angle $\alpha$ with the ambient field taken along the $z$-axis, it is convenient to perform the change of reference frame $x^{\prime}=x \cos \alpha-z \sin \alpha$ and $z^{\prime}=x \sin \alpha+z \cos \alpha$. We concentrate on perturbations depending only on the coordinate $z^{\prime}$ along the direction of propagation, that is stretched as $\xi=\epsilon^{1 / 2}\left(z^{\prime}-V_{0} t\right)$. The small parameter $\epsilon$ measures the amplitude of the magnetosonic waves that are selected by perturbing the equilibrium state in the form

$\rho=\rho^{(0)}+\epsilon \rho^{(1)}+\cdots, \quad p_{\perp r}=p_{\perp r}^{(0)}+\epsilon p_{\perp r}^{(1)}+\cdots$,

$p_{\| r}=p_{\| r}^{(0)}+\epsilon p_{\| r}^{(1)}+\cdots$,

$b_{z}=B_{0}+\epsilon b_{z}^{(1)}+\cdots, \quad b_{x}=\epsilon b_{x}^{(1)}+\cdots$,

$u_{z}=\epsilon u_{z}^{(1)}+\cdots, \quad u_{x}=\epsilon u_{x}^{(1)}+\cdots$,

$b_{y}=\epsilon^{3 / 2} b_{y}^{(1)}+\cdots, \quad u_{y}=\epsilon^{3 / 2} u_{y}^{(1)}+\cdots$

The dispersion and the nonlinearities then act on the slow time $\tau=\epsilon^{3 / 2} t$. The reductive perturbative expansion is straightforward and, in the absence of Landau damping, leads to the usual Korteweg-de Vries equation (Kakutani et al., 1968; Kever and Morikawa, 1969; Chakraborty and Das, 2000). In the regimes where the strength of the Landau damping is small enough to be comparable with dispersion and nonlinearity, for example in the case of hot electrons and cold ions, a Korteweg-de Vries equation with Landau damping is obtained (Janaki et al., 1992). We do not restrict ourselves here to this special regime and compute the Landau damping that arises at the level of the linear dispersion relation.

From the induction and the density equations, one gets to leading order

$\frac{b_{x}^{(1)}}{B_{0}}+\cos \alpha \frac{u_{x}^{(1)}}{V_{0}}=0$

$\frac{b_{z}^{(1)}}{B_{0}}-\sin \alpha \frac{u_{x}^{(1)}}{V_{0}}=0$

$\sin \alpha \frac{u_{x}^{(1)}}{V_{0}}+\cos \alpha \frac{u_{z}^{(1)}}{V_{0}}-\frac{\rho^{(1)}}{\rho^{(0)}}=0$,

that leads to simple expressions in terms of the normalized magnetic perturbation $A$ defined by $\frac{|b|}{B_{0}}=1+\epsilon A+\cdots$. With the scaling associated with magnetosonic waves, one has $\frac{b_{z}^{(1)}}{B_{0}}=A$ and thus writes $\frac{b_{x}^{(1)}}{B_{0}}=-\frac{1}{\tan \alpha} A, \frac{u_{x}^{(1)}}{V_{0}}=\frac{1}{\sin \alpha} A$ and $\frac{u_{z}^{(1)}}{V_{0}}=\frac{1}{\cos \alpha}\left(\frac{\rho^{(1)}}{\rho^{(0)}}-A\right)$. The linearization of the pressure equations gives

$-\frac{p_{\| r}^{(1)}}{p_{\|}^{(0)}}+\sin \alpha \frac{u_{x}^{(1)}}{V_{0}}+3 \cos \alpha \frac{u_{z}^{(1)}}{V_{0}}+\cos \alpha \frac{q_{\| r}^{(1)}}{V_{0} p_{\| r}^{(0)}}=0$
$-\frac{p_{\perp r}^{(1)}}{p_{\perp}^{(0)}}+2 \sin \alpha \frac{u_{x}^{(1)}}{V_{0}}+\cos \alpha \frac{u_{z}^{(1)}}{V_{0}}+\cos \alpha \frac{q_{\perp r}^{(1)}}{V_{0} p_{\perp r}^{(0)}}=0$

that leads to

$$
\begin{aligned}
& q_{\| r}^{(1)}=\frac{V_{0} p_{\| r}^{(0)}}{\cos \alpha}\left(\frac{p_{\| r}^{(1)}}{p_{\| r}^{(0)}}-3 \frac{\rho^{(1)}}{\rho^{(0)}}+2 A\right) \\
& q_{\perp r}^{(1)}=\frac{V_{0} p_{\perp r}^{(0)}}{\cos \alpha}\left(\frac{p_{\perp r}^{(1)}}{p_{\perp r}^{(0)}}-\frac{\rho^{(1)}}{\rho^{(0)}}-A\right) .
\end{aligned}
$$

The closure equations provide

$$
\begin{aligned}
& \left(-V_{0}+\frac{v_{t h, r} \cos \alpha}{\sqrt{\frac{8}{\pi}}\left(1-\frac{3 \pi}{8}\right)} \mathcal{H}\right) \frac{q_{\| r}^{(1)}}{v_{t h, r} p_{\| r}^{(0)}}=\frac{v_{t h, r}}{1-\frac{3 \pi}{8}} \cos \alpha\left(\frac{p_{\| r}^{(1)}}{p_{\| r}^{(0)}}-\frac{\rho^{(1)}}{\rho^{(0)}}\right) \\
& \left(-V_{0}-\sqrt{\frac{\pi}{2}} v_{t h, r} \cos \alpha \mathcal{H}\right) \frac{q_{\perp r}^{(1)}}{v_{t h, r} p_{\perp r}^{(0)}}=-v_{t h, r} \cos \alpha\left(\frac{p_{\perp r}^{(1)}}{p_{\perp r}^{(0)}}-\frac{\rho^{(1)}}{\rho^{(0)}}+\left(\frac{T_{\perp r}^{(0)}}{T_{\| r}^{(0)}}-1\right) A\right) .
\end{aligned}
$$

After substitution, one easily expresses the transverse pressure perturbations as where $c_{r}=\frac{V_{0}}{v_{t h, r} \cos \alpha}$ and

$$
\mathcal{W}_{2}\left(c_{r}\right)=\frac{1}{1-\sqrt{\frac{\pi}{2}} c_{r} \mathcal{H}-c_{r}^{2}}
$$

$\frac{p_{\perp r}^{(1)}}{p_{\perp r}^{(0)}}=\frac{\rho^{(1)}}{\rho^{(0)}}+\left(1-\frac{T_{\perp r}^{(0)}}{T_{\| r}^{(0)}} \mathcal{W}_{2}\left(c_{r}\right)\right) A$ 
can be viewed as the two-pole approximant of the plasma response function

$\mathcal{W}\left(c_{r}\right)=\frac{1}{\sqrt{2 \pi}} \mathrm{P} \int \frac{\zeta e^{-\zeta^{2} / 2}}{\zeta-c_{r}} d \zeta+\sqrt{\frac{\pi}{2}} c_{r} e^{-c_{r}^{2} / 2} \mathcal{H}$
The parallel pressure perturbations involve the four-pole approximant of the plasma response function, in the form

$\mathcal{W}_{4}\left(c_{r}\right)=\frac{\frac{1}{2}(8-3 \pi) c_{r}^{2}-\sqrt{2 \pi} c_{r} \mathcal{H}+4}{\frac{1}{2} c_{r}^{4}(3 \pi-8)+\sqrt{2} c_{r}^{3} \mathcal{H}+\frac{1}{2}(16-9 \pi) c_{r}^{2}-3 \sqrt{2 \pi} c_{r} \mathcal{H}+4}$.

One has

$\frac{p_{\| r}^{(1)}}{p_{\| r}^{(0)}}=\left(c_{r}^{2}+\mathcal{W}_{4}^{-1}\left(c_{r}\right)\right) \frac{\rho^{(1)}}{\rho^{(0)}}-\left(c_{r}^{2}-1+\mathcal{W}_{4}^{-1}\left(c_{r}\right)\right) A$.

Turning to the momentum equation, one gets

$-V_{0}^{2} \frac{u_{x}^{(1)}}{V_{0}}+\frac{p_{\perp}^{(0)}}{\rho^{(0)}} \sin \alpha \frac{p_{\perp}^{(1)}}{p_{\perp}^{(0)}}+\left(\frac{p_{\|}^{(0)}}{\rho^{(0)}}-\frac{p_{\perp}^{(0)}}{\rho^{(0)}}\right) \cos \alpha \frac{b_{x}^{(1)}}{B_{0}}-\frac{B_{0}^{2}}{4 \pi \rho^{(0)}}\left(\cos \alpha \frac{b_{x}^{(1)}}{B_{0}}-\sin \alpha \frac{b_{z}^{(1)}}{B_{0}}\right)=0$

$-V_{0}^{2} \frac{u_{z}^{(1)}}{V_{0}}-\cos \alpha\left(\frac{p_{\|}^{(0)}}{\rho^{(0)}}-\frac{p_{\perp}^{(0)}}{\rho^{(0)}}\right) \frac{b_{z}^{(1)}}{B_{0}}+\cos \alpha \frac{p_{\|}^{(1)}}{\rho^{(0)}}=0$.

After substitution of the total parallel and perpendicular pressures $p_{\perp}^{(1)}=\sum_{r} p_{\perp r}^{(1)}$ and $p_{\|}^{(1)}=\sum_{r} p_{\| r}^{(1)}$, one obtains

$$
\begin{aligned}
& \frac{p_{\perp}^{(0)}}{\rho^{(0)}} \sin ^{2} \alpha \frac{\rho^{(1)}}{\rho^{(0)}}+\left[v_{A}^{2}-V_{0}^{2}+\left(\frac{p_{\perp}^{(0)}}{\rho^{(0)}}-\frac{p_{\|}^{(0)}}{\rho^{(0)}}\right) \cos ^{2} \alpha+\sin ^{2} \alpha \sum_{r} \frac{p_{\perp r}^{(0)}}{\rho^{(0)}}\left(1-\frac{T_{\perp r}^{(0)}}{T_{\| r}^{(0)}} \mathcal{W}_{2}\left(c_{r}\right)\right)\right] A=0 \\
& {\left[-V_{0}^{2}+\cos ^{2} \alpha \sum_{r} \frac{p_{\| r}^{(0)}}{\rho^{(0)}}\left(c_{r}^{2}+\mathcal{W}_{4}^{-1}\left(c_{r}\right)\right)\right] \frac{\rho^{(1)}}{\rho^{(0)}}+\left[V_{0}^{2}+\cos ^{2} \alpha\left[\frac{p_{\perp}^{(0)}}{\rho^{(0)}}-\sum_{r} \frac{p_{\| r}^{(0)}}{\rho^{(0)}}\left(c_{r}^{2}+\mathcal{W}_{4}^{-1}\left(c_{r}\right)\right)\right]\right] A=0}
\end{aligned}
$$

where the Alfvén wave velocity $v_{A}$ is defined by $v_{A}^{2}=\frac{B_{0}^{2}}{4 \pi \rho^{(0)}}$.

The dispersion relation is then readily obtained. It is convenient to introduce the parameters $v_{\perp r}^{2}=\frac{p_{\perp r}^{(0)}}{\rho^{(0)}}, v_{\| r}^{2}=\frac{p_{\| r}^{(0)}}{\rho^{(0)}}$, $v_{\perp}^{2}=\sum_{r} v_{\perp r}^{2}, \quad v_{\|}^{2}=\sum_{r} v_{\| r}^{2}$ and $v_{\Delta}^{2}=v_{\perp}^{2}-v_{\|}^{2}$ together with, following Ferrière and André (2002), the operators

$\mathcal{C}_{\|}^{2}=\sum_{r} v_{\| r}^{2}\left(c_{r}^{2}+\mathcal{W}_{4}^{-1}\left(c_{r}\right)\right)$

and

$\mathcal{C}_{\perp}^{2}=v_{\perp}^{2}+\sum_{r} v_{\perp r}^{2}\left(1-\frac{T_{\perp r}^{(0)}}{T_{\| r}^{(0)}} \mathcal{W}_{2}\left(c_{r}\right)\right)=2 v_{\perp}^{2}-\sum_{r} \frac{v_{\perp r}^{4}}{v_{\| r}^{2}} \mathcal{W}_{2}\left(c_{r}\right)$

One has

$$
\begin{aligned}
V_{0}^{4}-\left[\left(v_{A}^{2}+v_{\Delta}^{2}+\mathcal{C}_{\|}^{2}\right) \cos ^{2} \alpha+\left(v_{A}^{2}+\mathcal{C}_{\perp}^{2}\right) \sin ^{2} \alpha\right] V_{0}^{2} \\
+\left(\left(v_{A}^{2}+v_{\Delta}^{2}\right) \mathcal{C}_{\|}^{2} \cos ^{2} \alpha+\left[\left(v_{A}^{2}+\mathcal{C}_{\perp}^{2}\right) \mathcal{C}_{\|}^{2}-v_{\perp}^{4}\right] \sin ^{2} \alpha\right) \cos ^{2} \alpha=0,
\end{aligned}
$$

which, up to the replacements of the plasma response function by suitable Padé approximants, identifies with the dispersion relation directly derived from the Vlasov-Maxwell system taken in the long-wave limit (Appendix A), or from a mixed fluid-kinetic formalism (Ferrière and André, 2002).

A simple expression for the Landau damping is obtained under the condition $v_{t h, p} \ll V_{0} \ll v_{t h, e}$, associated with cold protons (following an adiabatic equation of state) and hot electrons (in an isothermal state). In these limits, $c_{p} \gg 1$ and $\mathcal{W}\left(c_{p}\right) \approx-1 / c_{p}^{2}-3 / c_{p}^{4}-15 / c_{p}^{6}$, while $c_{e} \ll 1$ and $\mathcal{W}\left(c_{e}\right) \approx 1-c_{e}^{2}+\sqrt{\frac{\pi}{2}} c_{e} \mathcal{H}$. Note that the Padé approximants of the plasma response function are constructed in a way that reproduces the adiabatic and isothermal limits. As a consequence, the Landau-fluid predicts exact results in the considered regime. For the sake of simplicity and easy comparison with the literature, we assume isotropic 
temperatures for the equilibrium state. Taking into account the smallness of the ratio $m_{e} / m_{p}$ and also the assumption $\frac{m_{e}}{m_{p}} \ll \beta \ll \frac{T_{e}}{T_{p}}$ where $\beta=\frac{1}{v_{A}^{2}} \frac{T_{e}}{m_{p}}$ (that results from the condition $\left.v_{t h, p} \ll V_{0} \ll v_{t h, e}\right)$, one has $c_{e}=\sqrt{\frac{m_{e}}{m_{p}}} \frac{\lambda}{\cos \alpha} \frac{1}{\sqrt{\beta}}$ with $\lambda=\frac{V_{0}}{v_{A}}$. One then easily gets the following formulas valid in the case of a finite angle of propagation,

$\frac{\mathcal{C}_{\|}^{2}}{v_{A}^{2}}=\frac{\mathcal{C}_{\perp}^{2}}{v_{A}^{2}}=\beta\left(1-\sqrt{\frac{\pi}{2}} \sqrt{\frac{m_{e}}{m_{p}}} \frac{1}{\sqrt{\beta}} \frac{\lambda}{\cos \alpha} \mathcal{H}\right)$,

that are to be substituted in the dispersion relation

$\lambda^{4}-\lambda^{2}\left(1+\frac{\mathcal{C}_{\|}^{2}}{v_{A}^{2}} \cos ^{2} \alpha+\frac{\mathcal{C}_{\perp}^{2}}{v_{A}^{2}} \sin ^{2} \alpha\right)+\cos ^{2} \alpha\left[\frac{\mathcal{C}_{\|}^{2}}{v_{A}^{2}}+\sin ^{2} \alpha\left(\frac{\mathcal{C}_{\|}^{2} \mathcal{C}_{\perp}^{2}}{v_{A}^{4}}-\frac{p^{(0) 2}}{v_{A}^{4} \rho^{(0) 2}}\right)\right]=0$

One obtains

$\lambda^{4}-\lambda^{2}\left(1+\beta-\sqrt{\beta} \sqrt{\frac{\pi}{2}} \sqrt{\frac{m_{e}}{m_{p}}} \frac{\lambda}{\cos \alpha} \mathcal{H}\right)+\cos ^{2} \alpha\left[\beta-\sqrt{\beta} \sqrt{\frac{\pi}{2}} \sqrt{\frac{m_{e}}{m_{p}}} \frac{\lambda}{\cos \alpha}\left(1+2 \beta \sin ^{2} \alpha\right) \mathcal{H}\right]=0$.

A solution of the form $\lambda=\lambda_{R}+\lambda_{I} \mathcal{H}$ with $\lambda_{I} \ll \lambda_{R}$ satisfies to leading order

$\lambda_{R}^{4}-(1+\beta) \lambda_{R}^{2}+\beta \cos ^{2} \alpha=0$ or

$$
\lambda_{R}^{2}=\frac{1+\beta \pm \sqrt{(1+\beta)^{2}-4 \beta \cos ^{2} \alpha}}{2},
$$

with a dissipative part prescribed by

$2\left(2 \lambda_{R}^{2}-\beta-1\right) \lambda_{I}=\sqrt{\beta} \sqrt{\frac{\pi}{2}} \sqrt{\frac{m_{e}}{m_{p}}} \frac{1}{\cos \alpha}\left[-\lambda_{R}^{2}+\cos ^{2} \alpha\left(1+2 \beta \sin ^{2} \alpha\right)\right]$.

It is then convenient to write

$-\lambda_{R}^{2}+\cos ^{2} \alpha\left(1+2 \beta \sin ^{2} \alpha\right)=\left(-\lambda_{R}^{2}+\beta \cos ^{2} \alpha\right) \sin ^{2} \alpha-\left(\lambda_{R}^{2}-1-\beta \sin ^{2} \alpha\right) \cos ^{2} \alpha$.

Using the equation satisfied by $\lambda_{R}$, one has

$\lambda_{R}^{2}-1-\beta \sin ^{2} \alpha=-\frac{\beta^{2} \sin ^{2} \alpha \cos ^{2} \alpha}{-\lambda_{R}^{2}+\beta \cos ^{2} \alpha}$.

This enables one to write the normalized damping rate $\lambda_{I}$ in the form

$$
\begin{aligned}
\lambda_{I}=-\sqrt{\beta} \sqrt{\frac{\pi}{8}} \sqrt{\frac{m_{e}}{m_{p}}} \cdot \frac{\sin ^{2} \alpha}{\cos \alpha} \\
\cdot \frac{\left(\lambda_{R}^{2}-\beta \cos ^{2} \alpha\right)^{2}+\beta^{2} \cos ^{4} \alpha}{\left(2 \lambda_{R}^{2}-\beta-1\right)\left(\lambda_{R}^{2}-\beta \cos ^{2} \alpha\right)},
\end{aligned}
$$

that also rewrites

$$
\begin{aligned}
\lambda_{I}=-\sqrt{\beta} & \sqrt{\frac{\pi}{8}} \sqrt{\frac{m_{e}}{m_{p}}} \cdot \frac{\sin ^{2} \alpha}{\cos \alpha} \\
\cdot & {\left[1+\frac{2 \beta^{2} \cos ^{4} \alpha}{\left(1+\beta-2 \beta \cos ^{2} \alpha\right) \lambda_{R}^{2}-\beta \cos ^{2} \alpha}\right] . }
\end{aligned}
$$

Equation (38) reproduces Eq. (5.5.2.10) of Akhiezer et al. (1975) up to the factor $\frac{\sin ^{2} \alpha}{\cos \alpha}$ whose absence seems to be a misprint. This factor is present in Janiki et al. (1992), but is there multiplied by a different expression.

\section{Parallel Alfvén waves}

A long-wave perturbative expansion of the Landau-fluid model was performed in the case of parallel Alfvén waves by Passot and Sulem (2003b). The stretched coordinate along the propagation is $\xi=\epsilon\left(z^{\prime}-V_{0} t\right)$ and the perturbation amplitudes obeys the scalings $b_{x} \sim b_{y} \sim u_{x} \sim u_{y}=O\left(\epsilon^{1 / 2}\right)$ and $u_{z} \sim b_{z}-B_{0} \sim \rho-\rho^{(0)} \sim P-P^{(0)}=O(\epsilon)$, with a dynamical time $\tau=\epsilon^{3 / 2} t$. The FLR corrections play a role at the level of the wave dispersion coefficient. In the present regime, they can accurately be captured when using the approximation given in Yajima (1966) that, in a local frame where the $\mathrm{z}$-axis is taken along the local magnetic field, reads

$$
\begin{aligned}
& \Pi_{x x}^{[1]}=-\Pi_{y y}^{[1]}=-\frac{p_{\perp p}}{2 \Omega_{p}}\left(\partial_{y} u_{x}+\partial_{x} u_{y}\right) \\
& \Pi_{z z}^{[1]}=0 \\
& \Pi_{x y}^{[1]}=\Pi_{y x}^{[1]}=-\frac{p_{\perp p}}{2 \Omega_{p}}\left(\partial_{y} u_{y}-\partial_{x} u_{x}\right) \\
& \Pi_{y z}^{[1]}=\Pi_{z y}^{[1]}=\frac{1}{\Omega_{p}}\left[2 p_{\| p} \partial_{z} u_{x}+p_{\perp p}\left(\partial_{x} u_{z}-\partial_{z} u_{x}\right)\right] \\
& \Pi_{x z}^{[1]}=\Pi_{z x}^{[1]}=-\frac{1}{\Omega_{p}}\left[2 p_{\| p} \partial_{z} u_{y}+p_{\perp p}\left(\partial_{y} u_{z}-\partial_{z} u_{y}\right)\right]
\end{aligned}
$$


Here, $\Omega_{p}=\frac{q_{p} B_{0}}{c m_{p}}$ is the proton cyclotron frequency, and the superscript [1] in the FLR tensor components refers to the order of the expansion in terms of $1 / \Omega_{p}$. For parallel Alfvén waves, the Landau damping does not act as a linear process but rather affects the nonlinear coupling. A long-wave reductive perturbative expansion performed on the resulting Landau-fluid model reproduces the derivative nonlinear Schrödinger equation obtained by an equivalent asymptotics performed on the Vlasov-Maxwell system, up to the replacement of the response function of the plasma by suitable Padé approximants in Eqs. (18) and (22) for the pressure perturbations. This agreement also holds in the case of multidimensional Alfvén wave trains where mean fields involving an averaging along the direction of propagation are driven by the presence of transverse gradients (Passot and Sulem, 2003a).

\section{Oblique Alfvén waves}

Turning to the case of obliquely propagating Alfvén waves, the reductive perturbative expansion now involves the scalings $b_{y}=\epsilon^{1 / 2}\left(b_{y}^{(1)}+\epsilon b_{y}^{(2)} \cdots\right), u_{y}=\epsilon^{1 / 2}\left(u_{y}^{(1)}+\epsilon u_{y}^{(2)}+\cdots\right)$, while the same expansions as in Sect. 3 are used for the other fields. As previously, we also assume that the disturbances of the equilibrium state vary only along the direction of propagation whose coordinate is stretched as $\xi=\epsilon^{1 / 2}\left(z^{\prime}-V_{0} t\right)$. We also introduce the slow time $\tau=\epsilon^{3 / 2} t$.
To leading order $\epsilon$, the $y$-components of the induction and momentum equations give

$\frac{u_{y}^{(1)}}{V_{0}}=-\frac{1}{\cos \alpha} \frac{b_{y}^{(1)}}{B_{0}}$,

and prescribes the propagation velocity of the Alfvén waves as $V_{0}=\Lambda_{0} \cos \alpha$ with

$\Lambda_{0}^{2}=\frac{B_{0}^{2}}{4 \pi \rho_{0}}+\frac{p_{\perp}^{(0)}}{\rho_{0}}-\frac{p_{\|}^{(0)}}{\rho_{0}}=v_{A}^{2}+v_{\Delta}^{2}$,

where we used notations defined in Sect. 3 .

At order $\epsilon^{3 / 2}$, the magnetic field divergenceless implies

$b_{x}^{(1)}=-\frac{1}{\tan \alpha} b_{z}^{(1)}$

and the $x$-component of the induction equation

$u_{x}^{(1)}=\frac{\Lambda_{0}}{\tan \alpha} \frac{b_{z}^{(1)}}{B_{0}}-\frac{\left(v_{A}^{2}+v_{\Delta e}^{2}\right)}{\Omega_{p}} \cos \alpha \partial_{\xi} \frac{b_{y}^{(1)}}{B_{0}}$.

Furthermore, the density equation gives

$u_{z}^{(1)}=\Lambda_{0} \frac{\rho^{(1)}}{\rho^{(0)}}-\Lambda_{0} \frac{b_{z}^{(1)}}{B_{0}}+\frac{\left(v_{A}^{2}+v_{\Delta e}^{2}\right)}{\Omega_{p}} \sin \alpha \partial_{\xi} \frac{b_{y}^{(1)}}{B_{0}}$.

The $x$ and $z$-components of the momentum equation express the pressure perturbations as

$$
\begin{array}{r}
\frac{p_{\perp}^{(1)}}{\rho^{(0)}}=\Lambda_{0} \frac{1}{\tan \alpha} u_{x}^{(1)}+v_{\Delta}^{2} \frac{1}{\tan \alpha} \frac{b_{x}^{(1)}}{B_{0}}+\frac{v_{\perp p}^{2}}{2 \Omega_{p}} \sin \alpha \partial_{\xi} u_{y}^{(1)}-\frac{v_{\perp p}^{2}-2 v_{\| p}^{2}}{\Omega_{p}} \frac{\cos ^{2} \alpha}{\sin \alpha} \partial_{\xi} u_{y}^{(1)} \\
+v_{A}^{2}\left(\frac{\cos \alpha}{\sin \alpha} \frac{b_{x}^{(1)}}{B_{0}}-\frac{b_{z}^{(1)}}{B_{0}}\right)-v_{A}^{2} \frac{b_{y}^{(1) 2}}{2 B_{0}^{2}}
\end{array}
$$

$\frac{p_{\|}^{(1)}}{\rho^{(0)}}=\Lambda_{0} u_{z}^{(1)}+v_{\Delta}^{2} \frac{\sin \alpha}{\cos \alpha} \frac{b_{x}^{(1)}}{B_{0}}-\frac{v_{\perp p}^{2}-2 v_{\| p}^{2}}{\Omega_{p}} \sin \alpha \partial_{\xi} u_{y}^{(1)}-\left(v_{A}^{2}+2 v_{\Delta}^{2}\right) \frac{b_{y}^{(1) 2}}{2 B_{0}^{2}}$,

that also rewrite as linear functions of $\rho^{(1)}, A=\frac{b_{z}^{(1)}}{B_{0}}+\frac{b_{y}^{(1) 2}}{2 B_{0}^{2}}$ and $\partial_{\xi} b_{y}^{(1)}$, in the form

$\frac{p_{\perp}^{(1)}}{\rho^{(0)}}=-v_{A}^{2} A-\frac{\Lambda_{0}}{\Omega_{p}}\left[\left(v_{A}^{2}+v_{\Delta e}^{2}-v_{\Delta p}^{2}+v_{\| p}^{2}\right) \frac{\cos ^{2} \alpha}{\sin \alpha}+\frac{v_{\perp p}^{2}}{2} \sin \alpha\right] \partial_{\xi} \frac{b_{y}^{(1)}}{B_{0}}$
$\frac{p_{\|}^{(1)}}{\rho^{(0)}}=\Lambda_{0}^{2} \frac{\rho^{(1)}}{\rho^{(0)}}-\left(v_{A}^{2}+2 v_{\Delta}^{2}\right) A+\frac{\Lambda_{0}}{\Omega_{p}}\left(v_{A}^{2}+v_{\Delta e}^{2}+v_{\Delta p}^{2}-v_{\| p}^{2}\right) \sin \alpha \partial_{\xi} \frac{b_{y}^{(1)}}{B_{0}}$.

At order $\epsilon^{2}$, the $y$-component of the momentum equation requires an accurate description of the FLR corrections. The usual approximation (40)-(44) is not sufficient and the re- quired corrections, expressed by the tensor $\Pi^{[2]}$, are computed in Appendix B. Together with the induction equation at the same order, one then obtains

$$
\begin{aligned}
V_{0}^{2}\left[\cos \alpha \partial_{\xi} \frac{u_{y}^{(2)}}{V_{0}}+\partial_{\xi} \frac{b_{y}^{(2)}}{B_{0}}\right]=\cos \alpha \partial_{\tau} u_{y}^{(1)}-\cos \alpha \partial_{\xi}\left[v_{\Delta}^{2}\left(\sin \alpha \frac{b_{x}^{(1)}}{B_{0}}-\cos \alpha \frac{b_{z}^{(1)}}{B_{0}}\right) \frac{b_{y}^{(1)}}{B_{0}}\right. \\
\left.+\cos \alpha\left(\frac{p_{\perp}^{(1)}}{\rho^{(0)}}-\frac{p_{\|}^{(1)}}{\rho^{(0)}}\right) \frac{b_{y}^{(1)}}{B_{0}}-v_{\Delta}^{2} \cos \alpha \frac{b_{y}^{(1) 3}}{B_{0}^{3}}\right]+\frac{\cos \alpha}{\Omega_{p}}\left(\frac{v_{\perp p}^{2}}{2} \sin ^{2} \alpha+2 v_{\| p}^{2} \cos ^{2} \alpha\right) \partial_{\xi \xi} u_{x}^{(1)}
\end{aligned}
$$




$$
\begin{gathered}
+\frac{1}{\Omega_{p}} v_{\perp p}^{2} \cos ^{2} \alpha\left(\sin \alpha \partial_{\xi \xi} u_{z}^{(1)}-\cos \alpha \partial_{\xi \xi} u_{x}^{(1)}\right)-\frac{1}{\rho^{(0)}} \partial_{\xi}\left(\sin \alpha \Pi_{x y}^{[2]}+\cos \alpha \Pi_{y z}^{[2]}\right) \\
V_{0}\left[\cos \alpha \partial_{\xi} \frac{u_{y}^{(2)}}{V_{0}}+\partial_{\xi} \frac{b_{y}^{(2)}}{B_{0}}\right]=\partial_{\tau} \frac{b_{y}^{(1)}}{B_{0}}+\cos \alpha \partial_{\xi}\left(u_{z}^{(1)} \frac{b_{y}^{(1)}}{B_{0}}\right)+\sin \alpha \partial_{\xi}\left(u_{x}^{(1)} \frac{b_{y}^{(1)}}{B_{0}}\right) \\
+\frac{v_{A}^{2}}{\Omega_{p}} \cos \alpha \partial_{\xi \xi}\left(\cos \alpha \frac{b_{x}^{(1)}}{B_{0}}-\sin \alpha \frac{b_{z}^{(1)}}{B_{0}}\right)+\frac{v_{\Delta e}^{2}}{\Omega_{p}} \partial_{\xi \xi}\left[\left(\cos ^{2} \alpha-\sin ^{2} \alpha\right) \frac{b_{x}^{(1)}}{B_{0}}+2 \sin \alpha \cos \alpha \frac{b_{y}^{(1) 2}}{2 B_{0}^{2}}\right] \\
+\frac{1}{\Omega_{p}} \cos \alpha \sin \alpha \partial_{\xi \xi} \frac{p_{\| e}^{(1)}-p_{\perp e}^{(1)}}{\rho^{(0)}} .
\end{gathered}
$$

The correction term $\Pi^{[2]}$ is due to the distortion of the magnetic field lines, to the time derivative of $\Pi^{[1]}$ and also to the contributions of the Hall effect and of the electron pressure gradient. One has

$$
\begin{aligned}
\frac{1}{\rho^{(0)}} \partial_{\xi}\left(\sin \alpha \Pi_{x y}^{[2]}\right. & \left.+\cos \alpha \Pi_{y z}^{[2]}\right)=2 \sin \alpha \cos \alpha \frac{\Lambda_{0}}{\Omega_{p}}\left(v_{\| p}^{2}-v_{\perp p}^{2}\right) \partial_{\xi \xi}\left(\frac{b_{y}^{(1) 2}}{2 B_{0}^{2}}\right) \\
+ & \frac{1}{\Omega_{p}^{2}}\left[-\Lambda_{0}^{2}\left(2 v_{\| p}^{2}-v_{\perp p}^{2}\right) \cos ^{3} \alpha-\frac{\Lambda_{0}^{2}}{4} v_{\perp p}^{2} \cos \alpha \sin ^{2} \alpha-\left(v_{A}^{2}+v_{\Delta e}^{2}\right) v_{\Delta p}^{2} \cos ^{3} \alpha\right] \partial_{\xi \xi \xi} \frac{b_{y}^{(1)}}{B_{0}} .
\end{aligned}
$$

The corresponding solvability condition reads

$$
\begin{aligned}
2 \Lambda_{0} \partial_{\tau} \frac{b_{y}^{(1)}}{B_{0}}- & \frac{\Lambda_{0}}{\tan \alpha} \frac{\left(v_{A}^{2}+v_{\Delta e}^{2}\right)}{\Omega_{p}} \partial_{\xi \xi} A+\frac{1}{\Omega_{p}} \frac{\Lambda_{0}}{\tan \alpha}\left[\left(\frac{v_{\perp p}^{2}}{2}+2 v_{\Delta e}^{2}\right) \sin ^{2} \alpha+\left(v_{\perp p}^{2}-2 v_{\| p}^{2}\right) \cos ^{2} \alpha\right] \partial_{\xi \xi} A \\
& -\frac{\Lambda_{0}}{\Omega_{p}} v_{\perp p}^{2} \sin \alpha \cos \alpha \partial_{\xi \xi} \frac{\rho^{(1)}}{\rho^{(0)}}-\frac{\left(v_{A}^{2}+v_{\Delta e}^{2}\right)}{\Omega_{p}^{2}} \cos \alpha\left[\frac{v_{\perp p}^{2}}{2} \sin ^{2} \alpha+\left(v_{\perp p}^{2}-2 v_{\| p}^{2}\right) \cos ^{2} \alpha\right] \partial_{\xi \xi \xi} \frac{b_{y}^{(1)}}{B_{0}} \\
& -\frac{\left(v_{A}^{2}+v_{\Delta e}^{2}\right)}{\Omega_{p}^{2}}\left(v_{\perp p}^{2}-v_{\| p}^{2}\right) \cos ^{2} \alpha \partial_{\xi \xi \xi} \frac{b_{y}^{(1)}}{B_{0}}+\frac{\Lambda_{0}^{2}}{\Omega_{p}^{2}}\left[\frac{v_{\perp p}^{2}}{4} \sin ^{2} \alpha+\left(2 v_{\| p}^{2}-v_{\perp p}^{2}\right) \cos ^{2} \alpha\right] \partial_{\xi \xi \xi} \frac{b_{y}^{(1)}}{B_{0}} \\
& +\frac{\Lambda_{0}}{\Omega_{p}} \cos \alpha \sin \alpha \partial_{\xi \xi} \frac{p_{\| e}^{(1)}-p_{\perp e}^{(1)}}{\rho^{(0)}}=0 .
\end{aligned}
$$

At this point, one needs to express the perturbations of density, pressure and magnetic field as a function of the wave amplitude. These relations are a priori provided by the dynamical equations for the pressures and the heat fluxes. It however turns out that the scaling associated with oblique Alfvén waves invalidates the assumption that the non-gyrotropic parts of the heat flux tensor can be ignored in the equations for the gyrotropic pressure components. The gyrotropic components of the heat flux are indeed of order $\epsilon$ while the non-gyrotropic contributions involve the product of the equilibrium pressure with the current which is also of order $\epsilon$. This situation contrasts with that of oblique magnetosonic and parallel Alfvén waves for which $q_{\| r} \sim q_{\perp r}=O(\epsilon)$ while the current scales like $\epsilon^{3 / 2}$. We are thus led to restrict the present analysis to oblique Alfvén waves in a plasma with adiabatic protons and isothermal electrons, a regime where the above Landau fluid model remains valid. This regime associated with the condition $v_{t h, p} \ll v_{A} \ll v_{t h, e}$, corresponds to a situation where $\frac{m_{e}}{m_{p}} \ll \beta \ll \frac{T_{e}}{T_{p}}$.

In the adiabatic regime, all the heat flux components are zero so that we can use the equations governing the evolu- tion of the perpendicular and parallel pressures in the form

$$
\begin{aligned}
& \frac{p_{\perp r}^{(1)}}{p_{\perp r}^{(0)}}-\frac{\rho^{(1)}}{\rho^{(0)}}-A+\frac{v_{A}^{2}+v_{\Delta e}^{2}}{\Lambda_{0} \Omega_{p}} \sin \alpha \partial_{\xi} \frac{b_{y}^{(1)}}{B_{0}}=0 \\
& \frac{p_{\| r}^{(1)}}{p_{\| r}^{(0)}}-3 \frac{\rho^{(1)}}{\rho^{(0)}}+2 A-2 \frac{v_{A}^{2}+v_{\Delta e}^{2}}{\Lambda_{0} \Omega_{p}} \sin \alpha \partial_{\xi} \frac{b_{y}^{(1)}}{B_{0}}=0 .
\end{aligned}
$$

Assuming also isotropic temperatures for the unperturbed plasma, we get for the protons

$$
\begin{aligned}
& \frac{p_{\perp p}^{(1)}}{\rho^{(0)}}=\beta v_{A}^{2} \frac{T_{p}^{(0)}}{T_{e}^{(0)}}\left[\frac{\rho^{(1)}}{\rho^{(0)}}+A-\frac{v_{A}^{2}}{\Lambda_{0} \Omega_{p}} \sin \alpha \partial_{\xi} \frac{b_{y}^{(1)}}{B_{0}}\right] \\
& \frac{p_{\| p}^{(1)}}{\rho^{(0)}}=\beta v_{A}^{2} \frac{T_{p}^{(0)}}{T_{e}^{(0)}}\left[3 \frac{\rho^{(1)}}{\rho^{(0)}}-2 A+\frac{2 v_{A}^{2}}{\Lambda_{0} \Omega_{p}} \sin \alpha \partial_{\xi} \frac{b_{y}^{(1)}}{B_{0}}\right] .
\end{aligned}
$$

Concerning the equation of state for isothermal electrons, the relations obtained in the case of parallel propagation are expected to remain valid since the electrons move very rapidly (relatively to the propagation velocity of the wave) along the magnetic field lines in a way that equalizes temperatures, making the electron pressure insensitive to the propagation direction of the wave. Taking the isothermal limit 
$c_{e}=\frac{1}{\sqrt{\beta}} \frac{m_{e}}{m_{p}} \rightarrow 0$ for the electron plasma response function in Eqs. (18) and (22) (that can still be used in the present regime), we get

$$
\begin{aligned}
& \frac{p_{\perp e}^{(1)}}{\rho^{(0)}}=v_{A}^{2}\left[\beta \frac{\rho^{(1)}}{\rho^{(0)}}-\sqrt{\beta} \sqrt{\frac{\pi}{2}} \sqrt{\frac{m_{e}}{m_{p}}} \mathcal{H} A\right] \\
& \frac{p_{\| e}^{(1)}}{\rho^{(0)}}=v_{A}^{2}\left\{\left[\beta-\sqrt{\beta} \sqrt{\left.\frac{\pi}{2} \sqrt{\frac{m_{e}}{m_{p}}} \mathcal{H}\right] \frac{\rho^{(1)}}{\rho^{(0)}}}\right.\right.
\end{aligned}
$$

$$
\left.+\sqrt{\beta} \sqrt{\frac{\pi}{2}} \sqrt{\frac{m_{e}}{m_{p}}} \mathcal{H} A\right\} .
$$

We observe that $A$ and $\rho^{(1)}$ are linear functions of $\partial_{\xi} b_{y}^{(1)}$. We thus reach the conclusion that the solvability condition governing the evolution of $b_{y}^{(1)}$ does not include nonlinear terms and reduces to a linear Airy equation, as in the usual MHD description (Mio, Ogino, and Takeda, 1976; Gazol, Passot, and Sulem, 1998).

Substituting the total pressures in Eqs. (52) and (53), we obtain in the considered limit $\beta \frac{T_{p}}{T_{e}} \ll 1$,

$\beta\left(1+\frac{T_{p}}{T_{e}}\right) \frac{\rho^{(1)}}{\rho^{(0)}}+\left(1-\sqrt{\beta} \sqrt{\frac{\pi}{2}} \sqrt{\frac{m_{e}}{m_{p}}} \mathcal{H}\right) A=-\frac{v_{A}}{\Omega_{p}} \frac{\cos ^{2} \alpha}{\sin \alpha} \partial_{\xi} \frac{b_{y}^{(1)}}{B_{0}}$
$\left(\beta-1-\sqrt{\beta} \sqrt{\frac{\pi}{2}} \sqrt{\frac{m_{e}}{m_{p}}} \mathcal{H}\right) \frac{\rho^{(1)}}{\rho^{(0)}}+\left(1+\sqrt{\beta} \sqrt{\frac{\pi}{2}} \sqrt{\frac{m_{e}}{m_{p}}} \mathcal{H}\right) A=\frac{v_{A}}{\Omega_{p}} \sin \alpha \partial_{\xi} \frac{b_{y}^{(1)}}{B_{0}}$

whose solutions $\frac{\rho^{(1)}}{\rho^{(0)}}$ and $A$ are to be reported in the solvability condition (57).

Let us first neglect the Landau damping and compute

$\frac{\rho_{R}^{(1)}}{\rho^{(0)}}=-\frac{v_{A}}{\Omega_{p}} \frac{1}{\sin \alpha} \partial_{\xi} \frac{b_{y}^{(1)}}{B_{0}}$

$A_{R}^{(1)}=-\frac{v_{A}}{\Omega_{p}}\left(\frac{\cos ^{2} \alpha}{\sin \alpha}-\frac{\beta}{\sin \alpha}\right) \partial_{\xi} \frac{b_{y}^{(1)}}{B_{0}}$,

where the subscript $R$ refers to the non dissipative part. Substituting in Eq. (57), we simply get

$\partial_{\tau} \frac{b_{y}^{(1)}}{B_{0}}+\frac{v_{A}^{3}}{2 \Omega_{p}^{2}}\left(\cos ^{2} \alpha-\beta\right) \frac{\cos \alpha}{\sin ^{2} \alpha} \partial \xi \xi \xi \frac{b_{y}^{(1)}}{B_{0}}=0$, that identifies with the equation obtained in the framework of Hall-MHD for a plasma viewed as a polytropic gas (Mio et al., 1976; Gazol et al., 1998).

An explicit form of the dissipation rate due to Landau damping is easily obtained in the small $\beta$ limit. Writing $\rho^{(1)}=\rho_{R}^{(1)}+\mathcal{H} \rho_{I}^{(1)}$ and $A^{(1)}=A_{R}^{(1)}+\mathcal{H} A_{I}^{(1)}$, one gets to leading order

$$
\begin{aligned}
& A_{I}^{(1)}=\sqrt{\beta} \sqrt{\frac{\pi}{2}} \sqrt{\frac{m_{e}}{m_{p}}} A_{R}^{(1)} \\
& -\frac{\rho_{I}^{(1)}}{\rho^{(0)}}+A_{I}^{(1)}=\sqrt{\beta} \sqrt{\frac{\pi}{2}} \sqrt{\frac{m_{e}}{m_{p}}}\left(\frac{\rho_{R}^{(1)}}{\rho^{(0)}}-A_{R}^{(1)}\right) .
\end{aligned}
$$

which leads to the dynamical equation

$\partial_{\tau} \frac{b_{y}^{(1)}}{B_{0}}+\frac{v_{A}^{3}}{2 \Omega_{p}^{2}}\left[\frac{\cos ^{3} \alpha}{\sin ^{2} \alpha}+\sqrt{\beta \frac{\pi}{2} \frac{m_{e}}{m_{p}}} \cos ^{3} \alpha\left(\tan ^{2} \alpha+\frac{1}{\tan ^{2} \alpha}\right) \mathcal{H}\right] \partial_{\xi \xi \xi} \frac{b_{y}^{(1)}}{B_{0}}=0$,

that reproduces the Landau damping dissipation rate given by Akhiezer et al. (1975).

\section{Kinetic Alfvén waves}

When describing Alfvén waves propagating almost perpendicularly to the ambient field $(\cos \alpha \ll 1)$, a different ordering is to be used, namely $b_{y} \sim u_{y}=O\left(\epsilon^{1 / 2}\right), b_{z}=O(\epsilon)$, $b_{x} \sim u_{x} \sim u_{z}=O\left(\epsilon^{3 / 2}\right)$, together with $\partial_{x}=O\left(\epsilon^{1 / 2}\right)$, $\partial_{t}=-\Lambda_{0} \partial_{z} \sim \epsilon$ and $\partial_{\tau}=O\left(\epsilon^{2}\right)$. This corresponds to a propagation angle $\alpha$ such that $\cos \alpha=O\left(\epsilon^{1 / 2}\right)$. The longwave expansion also involves the contributions $u_{y}^{(2)}$ and $b_{y}^{(2)}$ arising at order $\epsilon^{3 / 2}$. The expansion leads to the following equations where the coordinates refer to the stretched ones)

$$
\begin{aligned}
& \frac{p_{\perp}^{(1)}}{\rho^{(0)}}-\frac{v_{\perp p}^{2}}{2 \Omega_{p}} \partial_{x} u_{y}^{(1)}+v_{A}^{2} A=0 \\
& \frac{p_{\|}^{(1)}}{\rho^{(0)}}-v_{\Delta}^{2} \partial_{z}^{-1} \partial_{x} \frac{b_{x}^{(1)}}{B_{0}}-\frac{\Lambda_{0}}{\Omega_{p}}\left(v_{\perp p}^{2}-2 v_{\| p}^{2}\right) \partial_{x} \frac{b_{y}^{(1)}}{B_{0}} \\
& +\left(v_{A}^{2}+2 v_{\Delta}^{2}\right) \frac{b_{y}^{(1) 2}}{2 B_{0}^{2}}=0 \\
& \partial_{t} u_{y}^{(1)}+\frac{p_{\|}^{(0)}-p_{\perp}^{(0)}}{\rho^{(0)}} \partial_{z} \frac{b_{y}^{(1)}}{B_{0}}-v_{A}^{2} \partial_{z} \frac{b_{y}^{(1)}}{B_{0}}=0 \\
& \partial_{t} \frac{b_{y}^{(1)}}{B_{0}}-\partial_{z} u_{y}^{(1)}=0
\end{aligned}
$$


$\partial_{t} \rho^{(1)}+\rho^{(0)} \partial_{x} u_{x}^{(1)}=0$

$\partial_{t} \frac{b_{z}^{(1)}}{B_{0}}+\partial_{x} u_{x}=-\frac{v_{A}^{2}+v_{\Delta e}^{2}}{\Omega_{p}} \partial_{x z} \frac{b_{y}^{(1)}}{B_{0}}$ $\partial_{x} b_{x}^{(1)}+\partial_{z} b_{z}^{(1)}=0$

$$
\begin{aligned}
& \partial_{t} u_{y}^{(2)}- \Lambda_{0}^{2} \partial_{z} \frac{b_{y}^{(2)}}{B_{0}}+\partial_{\tau} u_{y}^{(1)}-\Lambda_{0} \frac{\rho^{(1)}}{\rho^{(0)}} \partial_{z} u_{y}^{(1)}+u_{x}^{(1)} \partial_{x} u_{y}^{(1)}-v_{\Delta}^{2} \partial_{x}\left(\frac{b_{x}^{(1)} b_{y}^{(1)}}{B_{0}^{2}}\right) \\
&+ \partial_{z}\left(\frac{p_{\|}^{(1)}-p_{\perp}^{(1)}}{\rho^{(0)}} \frac{b_{y}^{(1)}}{B_{0}}+v_{\Delta}^{2} \frac{b_{y}^{(1) 3}}{B_{0}^{3}}+v_{\Delta}^{2} \frac{b_{y}^{(1)} b_{z}^{(1)}}{B_{0}^{2}}\right) \\
&+ \frac{v_{\perp p}^{2}}{2 \Omega_{p}} \partial_{x x} v_{x}-v_{A}^{2} \frac{b_{x}^{(1)}}{B_{0}} \partial_{x} \frac{b_{y}^{(1)}}{B_{0}}-v_{A}^{2} \frac{b_{z}^{(1)}}{B_{0}} \partial_{z} \frac{b_{y}^{(1)}}{B_{0}}-2 \frac{v_{\perp p}^{2}}{\Omega_{p}} \Lambda_{0} \partial_{x z} \frac{b_{y}^{(1) 2}}{2 B_{0}^{2}}+\frac{\Lambda_{0}}{4 \Omega_{p}^{2}} v_{\perp p}^{2} \partial_{z x x} u_{y}^{(1)}=0 \\
& \partial_{t} \frac{b_{y}^{(2)}}{B_{0}}-\partial_{z} u_{y}^{(2)}+\partial_{\tau} \frac{b_{y}^{(1)}}{B_{0}}-\partial_{z}\left(u_{y}^{(1)} \frac{b_{z}^{(1)}}{B_{0}}\right)+\partial_{x}\left(u_{x} \frac{b_{y}^{(1)}}{B_{0}}-u_{y}^{(1)} \frac{b_{y}^{(1)}}{B_{0}}\right)-\frac{v_{A}^{2}}{\Omega_{p}} \partial_{x z} \frac{b_{z}^{(1)}}{B_{0}} \\
&-\frac{v_{\Delta e}^{2}}{\Omega_{p}}\left(\partial_{x x} \frac{b_{x}^{(1)}}{B_{0}}-2 \partial_{x z} \frac{b_{y}^{(1) 2}}{2 B_{0}^{2}}\right)+\frac{1}{\Omega_{p}} \partial_{x z} \frac{p_{\| e}^{(1)}-p_{\perp e}^{(1)}}{\rho^{(0)}}=0,
\end{aligned}
$$

with $\partial_{z}\left(u_{y}^{(1)} \frac{b_{z}^{(1)}}{B_{0}}\right)-\partial_{x}\left(u_{y}^{(1)} \frac{b_{y}^{(1)}}{B_{0}}\right)=0$, since the dynamics is assumed one-dimensional $\left(\partial_{x}=\sin \alpha \partial_{\xi}\right.$ and $\left.\partial_{z}=\cos \alpha \partial_{\xi}\right)$. Using $\partial_{t}=-\Lambda_{0} \partial_{z}$, this leads to $v_{y}^{(1)}=-\Lambda_{0} \frac{b_{y}^{(1)}}{B_{0}}$, and the solvability condition reads

$$
\begin{aligned}
2 \Lambda_{0} \partial_{\tau} \frac{b_{y}^{(1)}}{B_{0}} & -\partial_{z}\left[\left(\frac{p_{\|}^{(1)}-p_{\perp}^{(1)}}{\rho^{(0)}}+2 v_{\Delta}^{2} A\right) \frac{b_{y}^{(1)}}{B_{0}}\right]+\Lambda_{0} \partial_{x}\left(u_{x} \frac{b_{y}^{(1)}}{B_{0}}\right)-\frac{\Lambda_{0}}{\Omega_{p}} v_{A}^{2} \partial_{x z} \frac{b_{z}^{(1)}}{B_{0}} \\
& -\frac{\Lambda_{0}}{\Omega_{p}} v_{\Delta e}^{2}\left(\partial_{x x} \frac{b_{x}^{(1)}}{B_{0}}-2 \partial_{x z} \frac{b_{y}^{(1) 2}}{2 B_{0}^{2}}\right)+\frac{\Lambda_{0}}{\Omega_{p}} \partial_{x z} \frac{p_{\| e}^{(1)}-p_{\perp e}^{(1)}}{\rho^{(0)}} \\
& -\frac{v_{\perp p}^{2}}{2 \Omega_{p}} \partial_{x x} u_{x}^{(1)}-2 \frac{\Lambda_{0}}{\Omega_{p}}\left(v_{\| p}^{2}-v_{\perp p}^{2}\right) \partial_{x z} \frac{b_{y}^{(1) 2}}{2 B_{0}^{2}}+\frac{\Lambda_{0}^{2}}{4 \Omega_{p}^{2}} v_{\perp p}^{2} \partial_{x x z} \frac{b_{y}^{(1)}}{B_{0}}=0 .
\end{aligned}
$$

One then computes

$$
\begin{aligned}
& \frac{p_{\|}^{(1)}}{\rho^{(0)}}-\frac{p_{\perp}^{(1)}}{\rho^{(0)}}+2 v_{\Delta}^{2} A= \\
& \quad \frac{\Lambda_{0}}{2 \Omega_{p}}\left(3 v_{\perp p}^{2}-4 v_{\| p}^{2}\right) \partial_{x} \frac{b_{y}^{(1)}}{B_{0}}+\Lambda_{0}^{2} \frac{b_{z}^{(1)}}{B_{0}}
\end{aligned}
$$

and uses Eq. (78) to express $u_{x}^{(1)}$. One also uses the assumption of one-dimensionality to write

$\Lambda_{0} \partial_{x}\left(u_{x}^{(1)} \frac{b_{y}^{(1)}}{B_{0}}\right)=$

$$
-\frac{\Lambda_{0}}{\Omega_{p}}\left(v_{A}^{2}+v_{\Delta e}^{2}\right) \partial_{x z} \frac{b_{y}^{(1) 2}}{2 B_{0}^{2}}+\Lambda_{0}^{2} \partial_{z} \frac{b_{y}^{(1)} b_{z}^{(1)}}{B_{0}^{2}}
$$

In the equation resulting from the substitution of the above quantities in Eq. (81), one eliminates $b_{z}^{(1)}$ by means of Eqs. (76) and (78) to get

$\Lambda_{0} \frac{b_{z}^{(1)}}{B_{0}}=\Lambda_{0} \frac{\rho^{(1)}}{\rho^{(0)}}-\frac{v_{A}^{2}+v_{\Delta e}^{2}}{\Omega_{p}} \partial_{x z} \frac{b_{y}^{(0)}}{B_{0}}$,

which leads to

$2 \Lambda_{0} \partial_{\tau} \frac{b_{y}^{(1)}}{B_{0}}+\frac{\Lambda_{0}}{2 \Omega_{p}}\left(v_{\perp p}^{2}-2\left(v_{A}^{2}-v_{\Delta e}^{2}\right)\right) \partial_{x z} A-\frac{\Lambda_{0}}{\Omega_{p}} v_{\perp p}^{2} \partial_{x z} \frac{\rho^{(1)}}{\rho^{(0)}}$

$$
+\frac{v_{\perp p}^{2}}{4 \Omega_{p}^{2}}\left(\Lambda_{0}^{2}-2\left(v_{A}^{2}+v_{\Delta e}^{2}\right)\right) \partial_{x x z} \frac{b_{y}^{(1)}}{B_{0}}+\frac{\Lambda_{0}}{\Omega_{p}} \partial_{x z} \frac{p_{\| e}^{(1)}-p_{\perp e}^{(1)}}{\rho^{(0)}}=0 .
$$


As in the previous section, we shall make use of the pressure equations in order to express $\rho^{(1)} / \rho^{(0)}$ and $A$. In Eq. (73) for the parallel pressure, we express $b_{y}^{(1) 2} / B_{0}^{2}$ in terms of $A$ and $b_{z}^{(1)} / B_{0}$ that is given by Eq. (84). This reproduces Eq. (52) where $\sin \alpha \partial_{\xi}$ is here denoted $\partial_{x}$. Equation (72) for the perpendicular pressure, together with the relation
$u_{y}^{(1)}=-\Lambda_{0} \frac{b_{y}^{(1)}}{B_{0}}$ is the same as Eq. (53) up to the $\frac{\cos ^{2} \alpha}{\sin \alpha}$ term that is subdominant in the kinetic Alfvén wave asymptotics. Assuming as previously adiabatic protons and isothermal electrons together with the hypothesis of isotropic temperatures for the equilibrium state, we get

$$
\begin{aligned}
& \beta\left(1+\frac{T_{p}^{(0)}}{T_{e}^{(0)}}\right) \frac{\rho^{(1)}}{\rho^{(0)}}+\left(1+\beta \frac{T_{p}^{(0)}}{T_{e}^{(0)}}\right) A-\sqrt{\beta} \sqrt{\frac{\pi}{2} \sqrt{\frac{m_{e}}{m_{p}}}} \mathcal{H} A=\frac{v_{A}}{2 \Omega_{p}} \beta \frac{T_{p}^{(0)}}{T_{e}^{(0)}} \partial_{x} \frac{b_{y}^{(1)}}{B_{0}} \\
& \left(-1+\beta+3 \beta \frac{T_{p}^{(0)}}{T_{e}^{(0)}}\right) \frac{\rho^{(1)}}{\rho^{(0)}}+\left(1-2 \beta \frac{T_{p}^{(0)}}{T_{e}^{(0)}}\right) A+\sqrt{\beta} \sqrt{\frac{\pi}{2}} \sqrt{\frac{m_{e}}{m_{p}}} \mathcal{H}\left(A-\frac{\rho^{(1)}}{\rho^{(0)}}\right)=\frac{v_{A}}{\Omega_{p}}\left(1-3 \beta \frac{T_{p}^{(0)}}{T_{e}^{(0)}}\right) \partial_{x} \frac{b_{y}^{(1)}}{B_{0}} .
\end{aligned}
$$

When neglecting the Landau damping, this system is solved as

$$
\begin{aligned}
& \frac{\rho^{(1)}}{\rho^{(0)}}=-\frac{v_{A}}{\Omega_{p}}\left(1-\frac{3}{2} \beta \frac{T_{p}^{(0)}}{T_{e}^{(0)}}\right) \partial_{x} \frac{b_{y}^{(1)}}{B_{0}} \\
& A=\frac{v_{A}}{\Omega_{p}} \beta\left(1+\frac{3}{2} \frac{T_{p}^{(0)}}{T_{e}^{(0)}}\right) \partial_{x} \frac{b_{y}^{(1)}}{B_{0}} .
\end{aligned}
$$

In the small $\beta$ limit, the Landau damping in Eq. (85) is due to the anisotropy of the electron pressure fluctuations, where the contribution of the density fluctuations is dominant. This leads to the equation governing the dynamics of long kinetic Alfvén waves

$$
\begin{aligned}
& \partial_{\tau} \frac{b_{y}^{(1)}}{B_{0}}+\frac{v_{A}^{3}}{2 \Omega_{p}^{2}} \cos \alpha {\left[-\beta\left(1+\frac{3}{4} \frac{T_{p}^{(0)}}{T_{e}^{(0)}}\right)\right.} \\
&\left.+\sqrt{\beta} \sqrt{\frac{\pi}{2}} \sqrt{\frac{m_{e}}{m_{p}}} \mathcal{H}\right] \partial_{\xi \xi \xi} \frac{b_{y}^{(1)}}{B_{0}}=0,
\end{aligned}
$$

where we made the substitution $\partial_{x x z}=\cos \alpha \partial_{\xi \xi \xi}$, the variable $\xi$ denoting the coordinate along the direction of propagation. Note that this equation is linear. It is valid for propagation angles $\alpha$ such that $\cos ^{2} \alpha \ll \beta \ll 1$. We recover in particular the effect on the dispersion of the protonelectron temperature ratio predicted by Hasegawa and Chen (1976) using a fully kinetic theory. The same dispersion coefficient was also obtained by Belmont and Rezeau (1987) using a bi-fluid approach. We here demonstrate that a onefluid approach can reproduce the correct dispersion. As already mentioned, the extension of the present approach to hotter plasmas and the derivation of the dispersion and dissipation of kinetic Alfvén waves in this regime (Lysak and Lotko, 1996) requires a specific modeling of the influence of the Hall effect on the heat fluxes and will be addressed in a forthcoming paper.

In a kinetic Alfvén wave, protons move perpendicularly to the average magnetic field while electrons move mainly along the magnetic field lines. As a consequence a parallel electric field $E_{\|}$develops which accelerates electrons. This parallel electric field is easily obtained from the generalized Ohm's law and, to leading order, read

$E_{\|}=-\frac{m_{p} c}{q_{p} \rho^{(0)}} \partial_{z}\left(n^{(1)} T_{e}\right)$.

Using that $E_{\perp} \approx v_{A} b_{y}^{(1)}$ one recovers the usual relation (Lysak 1990)

$E_{\|}=\rho_{s}^{2} \partial_{z} \nabla_{\perp} \cdot E_{\perp}$

with $\rho_{s}^{2}=T_{e}^{(0)} /\left(m_{p} \Omega_{p}^{2}\right)$. As mentioned by Hollweg (1999), and seen on Eq. (88), a kinetic Alfvén wave becomes compressive as soon as the transverse wavenumber $k_{\perp}$ satisfies the condition $k_{\perp} v_{A} / \Omega_{p} \simeq 1$, i.e. for scales of the order of the ion inertial length. For low $\beta$ plasmas, this occurs even though the wave dispersion is negligible.

It is remarkable that kinetic Alfvén waves with frequencies $\omega \ll \Omega_{p}$, can be described by a single fluid generalized MHD formalism. This point was noted by Marchenko, Denton and Hudson (1996) who derived the linear dispersion relation for kinetic Alfvén waves ignoring Landau damping. The crucial ingredients are a generalized Ohm's law with Hall term and electron pressure gradient, FLR corrections up to order $1 / \Omega_{p}^{2}$ and, when retaining wave dissipation, an appropriate form for the electron Landau damping.

\section{Conclusions}

We have studied the dynamics of dispersive MHD waves propagating in a collisionless plasma permeated by a strong magnetic field, using a Landau fluid model that retains finite Larmor radius corrections to the gyrotropic pressures together with the Hall effect and the electron pressure gradient in a generalized Ohm's law. The predicted Landau damping of magnetosonic waves with a wavelength large compared to the ion inertial length accurately reproduces that derived by a long-wave asymptotics from the Vlasov-Maxwell system. It is also demonstrated that the dynamics of smallamplitude oblique Alfvén waves is linear, even in the case of quasi-transverse propagation (kinetic Alfvén waves). The 
dispersion and damping rates, computed in the regime of adiabatic ions and isothermal electrons often considered in the literature, also agrees with the results of the kinetic theory (Akhiezer et al., 1975). Addressing more general regimes would require an extension of the Landau fluid closure described in Sect. 2 by retaining the influence of the Hall effect on the heat fluxes. Another development concerns the description of FLR corrections. In instances, such as kinetic Alfvén waves, a description going beyond Yajima's (1966) formulas is required. It is obtained in the present paper by a perturbative expansion that correctly retains the effect of the magnetic curvature, a prerequisite to get the exact cancellation of nonlinear terms whose presence would have made the resulting long-wave equation mathematically ill-posed.

As mentioned in the Introduction, a one-fluid description appears to be an efficient tool to investigate both analytically and by means of numerical simulations, the regime of magnetohydrodynamic-wave turbulence, as it occurs both in space and laboratory plasmas. Among the questions to be addressed are the anisotropy of the cascade, the typical scale at which the cascade is arrested and the compared heating of the electron and proton populations. The role played by dispersion is of specific interest and motivates the development of a weak turbulence theory for Hall-MHD (Sahraoui, Belmont and Rezeau, 2003).

\section{Appendix A: Kinetic theory of magnetosonic waves}

We write the Vlasov-Maxwell equations in the form

$$
\begin{aligned}
& \partial_{t} f_{r}+\boldsymbol{v} \cdot \nabla f_{r}+\frac{q_{r}}{m_{r}}\left(\boldsymbol{e}+\frac{1}{c} \boldsymbol{v} \times \boldsymbol{b}\right) \cdot \nabla_{v} f_{r}=0 \\
& \frac{1}{c} \partial_{t} \boldsymbol{b}=-\nabla \times \boldsymbol{e} \\
& \nabla \times \boldsymbol{b}=\frac{4 \pi}{c} \sum_{r} q_{r} n_{r} \int \boldsymbol{v} f_{r} d^{3} \boldsymbol{v}+\frac{1}{c} \partial_{t} \boldsymbol{e} \\
& \nabla \cdot \boldsymbol{e}=4 \pi \sum_{r} q_{r} n_{r} \int f_{r} d^{3} \boldsymbol{v},
\end{aligned}
$$

where $f_{r}$ and $n_{r}$ are the distribution functions and the average number densities of the particles of species $r$ with charge $q_{r}$ and mass $m_{r}$. The displacement current $\frac{1}{c} \partial_{t} e$ turns out to be negligible in the present analysis.
As in Sect. 3, where $\alpha$ is the angle between the ambient magnetic field $B_{0} \widehat{z}$ (where $\widehat{z}$ is the unit vector pointing along the $z$-axis) and the direction of propagation of the wave, it is then convenient to perform the change of frame $x^{\prime}=x \cos \alpha-z \sin \alpha, z^{\prime}=x \sin \alpha+z \cos \alpha$, the dynamics being assumed independent of the $y$ variable. We then introduce the stretched variable $\xi=\epsilon^{1 / 2}\left(z^{\prime}-V_{0} t\right)$ where $V_{0} \ll c$ is the wave velocity in the $\mathrm{z}$ direction, together with the slow time $\tau=\epsilon^{3 / 2} t$. It follows that the spatial gradient rewrites $\nabla=\left(\epsilon^{1 / 2} \sin \alpha \partial_{\xi}, 0, \epsilon^{1 / 2} \cos \alpha \partial_{\xi}\right)$.

In order to select oblique magnetosonic waves, we write $b_{x}=\epsilon b_{x}^{(1)}+\cdots, \quad b_{y}=\epsilon^{3 / 2} b_{y}^{(1)}+\cdots$, $b_{z}=B_{0}+\epsilon b_{z}^{(1)}+\cdots$ and thus, from Eq. (A.2), $e_{x}=\epsilon^{3 / 2} e_{x}^{(1)}+\cdots, e_{y}=\epsilon e_{y}^{(1)}+\cdots, e_{z}=\epsilon^{3 / 2} e_{z}^{(1)}+\cdots$, with $\frac{V_{0}}{c} b_{x}^{(1)}=-\cos \alpha e_{y}^{(1)}, \quad \frac{V_{0}}{c} b_{y}^{(1)}=\cos \alpha e_{x}^{(1)}-\sin \alpha e_{z}^{(1)}$, $\frac{V_{0}}{c} b_{z}^{(1)}=\sin \alpha e_{y}^{(1)}$.

We also expand the distribution function in the form

$f_{r}=F_{r}^{(0)}+\epsilon\left(f_{r}^{(0)}+\epsilon^{1 / 2} f_{r}^{(1)}+\cdots\right)$

where $F_{r}^{(0)}$ denotes the equilibrium velocity distribution function, assumed rotationally symmetric around the direction of the ambient field and symmetric relatively to forward and backward velocities along this direction, thus excluding the presence of equilibrium drifts.

It is also convenient to express the velocity $v$ in a cylindrical coordinate system by defining the velocity space angle $\phi=\tan ^{-1}\left(v_{z} / v_{y}\right)$. One writes

$\boldsymbol{v}=\left(v_{x}=v_{\perp} \cos \phi, \quad v_{y}=v_{\perp} \sin \phi, \quad v_{z}=v_{\|}\right)$

and

$$
\begin{aligned}
\nabla_{v}=\left(\cos \phi \partial_{v_{\perp}}-\right. & \frac{\sin \phi}{v_{\perp}} \partial_{\phi}, \\
& \left.\sin \phi \partial_{v_{\perp}}+\frac{\cos \phi}{v_{\perp}} \partial_{\phi}, \quad \partial_{v_{\|}}\right)
\end{aligned}
$$

Furthermore, $\quad \frac{q_{r}}{c m_{r}}\left(\boldsymbol{v} \times B_{0} \widehat{\boldsymbol{z}}\right) \cdot \nabla_{v}=-\Omega_{r} \partial_{\phi}, \quad$ where $\Omega_{r}=\frac{q_{r} B_{0}}{m_{r} c}$ is the cyclotron frequency of the particles of species $r$.

Expanding to the successive orders, one gets from Eq. (A.1),

$$
\begin{aligned}
\Omega_{r} \partial_{\phi} F_{r}^{(0)} & =0 \\
\Omega_{r} \partial_{\phi} f_{r}^{(0)}=\frac{q_{r}}{m_{r}}\left[\left(e_{y}^{(1)}+\frac{v_{\|} b_{x}^{(1)}}{c}\right) \sin \phi \partial_{v_{\perp}}-\frac{v_{\perp} b_{x}^{(1)}}{c} \sin \phi \partial_{v_{\|}}\right] F_{r}^{(0)} & \\
\Omega_{r} \partial_{\phi} f_{r}^{(1)}=\frac{q_{r}}{m_{r}}\left[\left(e_{x}^{(1)}-\frac{v_{\|} b_{y}^{(1)}}{c}\right) \cos \phi \partial_{v_{\perp}}+\left(e_{z}^{(1)}+\frac{v_{\perp} b_{y}^{(1)}}{c} \cos \phi\right) \partial_{v_{\|}}\right] F_{r}^{(0)} & +\left(\sin \alpha v_{\perp} \cos \phi+\cos \alpha v_{\|}-V_{0}\right) \partial_{\xi} f_{r}^{(0)} .
\end{aligned}
$$


Equation (A.8) indicates that $F_{r}^{(0)}$ is independent of the angle $\phi$. Equation (A.9) leads to

$f_{r}^{(0)}=\mathcal{D} F_{r}^{(0)} \cos \phi \frac{b_{x}^{(1)}}{B_{0}}+\bar{f}_{r}^{(0)}$

with $\mathcal{D}=\left(\Lambda_{0}-v_{\|}\right) \partial_{v_{\perp}}+v_{\perp} \partial_{v_{\|}}$and $\Lambda_{0}=V_{0} / \cos \alpha$. One denotes $\bar{f}_{r}^{(0)}=\left\langle f_{r}^{(0)}\right\rangle \equiv \frac{1}{2 \pi} \int f_{r}^{(0)} d \phi$.

The solvability condition of Eq. (A.10) together with the divergenceless condition for the magnetic field reads

$$
\begin{aligned}
\left(v_{\|}-\Lambda_{0}\right) \bar{f}_{r}^{(0)}= & -\left(v_{\|}-\Lambda_{0}\right) \frac{v_{\perp}}{2} \partial_{v_{\perp}} F_{r}^{(0)} A \\
& +\frac{v_{\perp}^{2}}{2} \partial_{v_{\|}} F_{r}^{(0)} A+\frac{q_{r}}{m_{r}} \partial_{v_{\|}} F_{r}^{(0)} \varphi
\end{aligned}
$$

where $A=\frac{b_{z}^{(1)}}{B_{0}}$ and the electric potential $\varphi$ is such that $e_{z}^{(1)}=-\partial_{z} \varphi=\cos \alpha \partial_{\xi} \varphi$. Finally, the $y$-component of Eq. (A.3) gives

$-\frac{1}{\sin \alpha} \partial_{\xi} A=\frac{4 \pi}{c B_{0}} \sum_{r} q_{r} n_{r} \int v_{\perp} \cos \phi \partial_{\phi} \widetilde{f}_{r}^{(1)} d^{3} v$.

Using Eq. (A.10), one obtains

$$
\begin{aligned}
-\frac{1}{\sin \alpha} \partial_{\xi} A & =-\frac{1}{v_{A}^{2}}\left(\Lambda_{0}^{2}-v_{\Delta}^{2}\right) \frac{\cos ^{2} \alpha}{\sin \alpha} \partial_{\xi} A \\
& +\frac{4 \pi}{B_{0}^{2}} \frac{\sin \alpha}{2} \partial_{\xi} \sum_{r} m_{r} n_{r} \int v_{\perp}^{2} \bar{f}_{r}^{(0)} d^{3} v .
\end{aligned}
$$

From Eq. (A.12), one has

$\sum_{r} m_{r} n_{r} \int v_{\perp}^{2} \bar{f}_{r}^{(0)} d^{3} v=4 p_{\perp}^{(0)} A+2 \mathcal{N} A+\mathcal{M} \varphi$

and from Eq. (A.4),

$\mathcal{M} A+\mathcal{L} \varphi=0$,

where

$$
\begin{aligned}
& \mathcal{L}=2 \pi \sum_{r} \frac{q_{r}^{2} n_{r}}{m_{r}} \int_{0}^{\infty} d\left(\frac{v_{\perp}^{2}}{2}\right) \mathcal{G}_{r} \\
& \mathcal{M}=2 \pi \sum_{r} q_{r} n_{r} \int_{0}^{\infty} d\left(\frac{v_{\perp}^{2}}{2}\right) \frac{v_{\perp}^{2}}{2} \mathcal{G}_{r} \\
& \mathcal{N}=2 \pi \sum_{r} m_{r} n_{r} \int_{0}^{\infty} d\left(\frac{v_{\perp}^{2}}{2}\right) \frac{v_{\perp}^{4}}{4} \mathcal{G}_{r},
\end{aligned}
$$

and

$$
\mathcal{G}_{r}=\mathrm{P} \int \frac{1}{v_{\|}-\Lambda_{0}} \frac{\partial F_{r}^{(0)}}{\partial v_{\|}} d v_{\|}+\left.\pi \frac{\partial F_{r}^{(0)}}{\partial v_{\|}}\right|_{v_{\|}=\Lambda_{0}} \mathcal{H}
$$

One finally gets

$$
\begin{gathered}
V_{0}^{2}-\left(v_{A}^{2}+v_{\Delta}^{2}\right) \cos ^{2} \alpha-\left(v_{A}^{2}+2 v_{\perp}^{2}+\frac{1}{\rho^{(0)}} \mathcal{N}\right) \\
\cdot \sin ^{2} \alpha+\frac{1}{\rho^{(0)}} \sin ^{2} \alpha \mathcal{M}^{2} \mathcal{L}^{-1}=0 .
\end{gathered}
$$

Assuming that the plasma contains only electrons and protons with bi-maxwellian equilibrium distribution functions

$$
F_{r}^{(0)}=\frac{1}{(2 \pi)^{3 / 2}} \frac{m_{r}^{3 / 2}}{T_{\perp r}^{(0)} T_{\| r}^{(0) 1 / 2}} \exp \left[-\frac{m_{r} v_{\|}^{2}}{2 T_{\| r}^{(0)}}-\frac{m_{r} v_{\perp}^{2}}{2 T_{\perp r}^{(0)}}\right]
$$

and using the quasi-neutrality condition of the equilibrium state $n_{p}^{(0)}=n_{e}^{(0)}=n^{(0)}$, one obtains

$$
\begin{gathered}
\mathcal{L}=\sum_{r}-n^{(0)} q_{r}^{2} \frac{1}{T_{\| r}^{(0)}} \mathcal{W}_{r} \\
\mathcal{M}=\sum_{r}-n^{(0)} q_{r} \frac{T_{\perp r}^{(0)}}{T_{\| r}^{(0)}} \mathcal{W}_{r} \\
\mathcal{N}=\sum_{r}-2 n^{(0)} \frac{T_{\perp r}^{(0) 2}}{T_{\| r}^{(0)}} \mathcal{W}_{r},
\end{gathered}
$$

where we define $\mathcal{W}_{r}=\mathcal{W}\left(c_{r}\right)$ with the notations of Sect. 3 .

Using Eq. (81) of Passot and Sulem (2003b) and introducing the notation $\mathcal{C}_{\perp}$ defined in Eq. (28), one recovers the dispersion relation of the magnetosonic waves given by Eq. (29).

\section{B: Second order FLR corrections to the pressure tensor}

In a regime where the electron inertia is negligible, the FLR corrections are relevant only for the proton pressure tensor $P_{p}$. They obey (Kulsrud, 1983)

$$
\begin{aligned}
\frac{\boldsymbol{b}}{B_{0}} \times \boldsymbol{\Pi}_{p}-\boldsymbol{\Pi}_{p} \times \frac{\boldsymbol{b}}{B_{0}} & =-\frac{1}{\Omega_{r}}\left[\frac{d \mathbf{P}_{p}}{d t}+\left(\nabla \cdot \boldsymbol{u}_{p}\right) \mathbf{P}_{p}\right. \\
& \left.+\mathbf{P}_{p} \cdot \nabla \boldsymbol{u}_{p}+\left(\mathbf{P}_{p} \cdot \nabla \boldsymbol{u}_{p}\right)^{t r}\right]
\end{aligned}
$$

where, as usual, the heat transfer contribution to the FLR have been neglected. To leading order, the local magnetic field $b$ is approximated by the ambient field in the left-handside and the ion pressure tensor $\mathbf{P}_{\mathbf{p}}$ in the right-hand-side is replaced by the gyrotropic contribution $\mathbf{P}_{\mathbf{p}}^{\mathbf{G}}$. This leads to the components of the leading order FLR correction tensor given by Eqs. (40)-(44). This approximation obtained in a local frame where the $z$-axis points along the local magnetic field is often oversimplified by using the same formula in a fixed frame with the $z$-axis along the ambient field (Khanna and Rajaram, 1982; Chakraborty and Das, 2000). These two references also involve a sign error in the definition of the components $\Pi_{x x}^{[1]}=-\Pi_{y y}^{[1]}$ and $\Pi_{x y}^{[1]}$. This approximation turns out to be insufficient in some instances, for example in the case of oblique Alfvén waves for which a higher order expansion is needed. For the scaling used in the description of oblique Alfvén waves for example, these corrections involve both terms of order $\epsilon$ and $\epsilon^{3 / 2}$. However they do not contain all the contributions arising at order $\epsilon^{3 / 2}$. In particular, one should also retain the effect of the magnetic field distortion, the fast-time derivative $\partial_{t} \Pi^{[1]}$, and also the Hall-term and electron pressure contribution to $\frac{d}{d t} \mathbf{P}_{\mathbf{r}}^{\mathbf{G}(\mathbf{1})}$. 
Denoting by $L$ (respectively $R$ ) the corrections of the left hand side (respectively right hand side) of Eq. (B.1) involving magnetic field distortion, one obtains

$$
\begin{aligned}
& 2 \Pi_{x y}^{[2]}+L_{x x}=\frac{1}{\Omega_{p}}\left(R_{x x}+\partial_{t} \Pi_{x x}^{[1]}+H_{x x}\right) \\
& \Pi_{y y}^{[2]}-\Pi_{x x}^{(2)}+L_{x y}=\frac{1}{\Omega_{p}}\left(R_{x y}+\partial_{t} \Pi_{x y}^{[1]}+H_{x y}\right) \\
& \Pi_{y z}^{[2]}+L_{x z}=\frac{1}{\Omega_{p}}\left(R_{x z}+\partial_{t} \Pi_{x z}^{[1]}+H_{x z}\right) \\
& -2 \Pi_{x y}^{[2]}+L_{y y}=\frac{1}{\Omega_{p}}\left(R_{y y}+\partial_{t} \Pi_{y y}^{[1]}+H_{y y}\right) \\
& -\Pi_{x z}^{[2]}+L_{y z}=\frac{1}{\Omega_{p}}\left(R_{y z}+\partial_{t} \Pi_{y z}^{[1]}+H_{y z}\right) \\
& L_{z z}=\frac{1}{\Omega_{p}}\left(R_{z z}+\partial_{t} \Pi_{z z}^{[1]}+H_{z z}\right),
\end{aligned}
$$

where the tensor $H$, associated with the Hall term and electron pressure contributions to the time variation of the direction of the magnetic field, is defined as

$\mathbf{H}=\frac{p_{\| r}-p_{\perp r}}{|\boldsymbol{b}|^{2}}\left(\boldsymbol{h}_{\boldsymbol{r}} \otimes \boldsymbol{b}+\boldsymbol{b} \otimes \boldsymbol{h}_{\boldsymbol{r}}-2 \frac{\boldsymbol{b} \otimes \boldsymbol{b}}{|\boldsymbol{b}|^{2}} \boldsymbol{b} \cdot \boldsymbol{h}_{\boldsymbol{r}}\right)$

with $\boldsymbol{h}_{\boldsymbol{r}}=\frac{c m_{r}}{q_{r}} \nabla \times\left(\frac{1}{4 \pi \rho}\left(\boldsymbol{b} \times(\nabla \times \boldsymbol{b})+\frac{1}{\rho} \nabla \cdot \mathbf{P}_{e}^{G}\right)\right.$.

For the specific case of oblique Alfvén waves for which the magnetic perturbation is dominated by $b_{y}$ (and under the assumption of no dependency in the $y$-coordinate), the nonzero contributions of the symmetric tensors $L, R$ and $H$ are given by

$$
\begin{aligned}
& L_{x x}=-2 \Pi_{x z}^{[1]} \frac{b_{y}}{B_{0}} \\
& L_{x y}=-\Pi_{y z}^{[1]} \frac{b_{y}}{B_{0}} \\
& L_{x z}=\left(\Pi_{x x}^{[1]}-\Pi_{z z}^{[1]}\right) \frac{b_{y}}{B_{0}} \\
& L_{y y}=0 \\
& L_{y z}=\Pi_{x y}^{[1]} \frac{b_{y}}{B_{0}} \\
& L_{z z}=2 \Pi_{x z}^{[1]} \frac{b_{y}}{B_{0}},
\end{aligned}
$$

and

$$
\begin{aligned}
& R_{x x}=p_{\perp r}^{(0)} \partial_{z} u_{y} \frac{b_{y}}{B_{0}} \\
& R_{y y}=\left(4 p_{\| r}^{(0)}-3 p_{\perp r}^{(0)}\right) \partial_{z} u_{y} \frac{b_{y}}{B_{0}} \\
& R_{y z}=\left(p_{\perp r}^{(0)} \partial_{x} u_{x}-2 p_{\| r}^{(0)} \partial_{z} u_{z}\right) \frac{b_{y}}{B_{0}} \\
& R_{z z}=-\left(4 p_{\| r}^{(0)}-2 p_{\perp r}^{(0)}\right) \partial_{z} u_{y} \frac{b_{y}}{B_{0}}
\end{aligned}
$$

together with

$$
H_{x z}=\frac{v_{A}^{2}+v_{\Delta_{e}}^{2}}{\Omega_{r}}\left(p_{\| p}^{(0)}-p_{\perp p}^{(0)}\right) \partial_{z z} \frac{b_{y}}{B_{0}} .
$$

Acknowledgements. This work benefited of support from CNRS programs "Soleil-Terre" and "Physique et Chimie du Milieu Interstellaire" and from INTAS contract 00-292.

Edited by: A. S. Sharma

Reviewed by: R. L. Lysak and another referee

\section{References}

Armstrong, J. W., Rickett, B. J., and Spangler, S. R.: Electron density power spectrum in the local interstellar medium, Astrophys. J., 443, 209-221, 1995.

Akhiezer, A. I., Akhiezer, I. A., Polovin, R. V., Sitenko, A. G., and Stepanov, K. N.: Plasma electrodynamics, Vol.1, Pergamon Press, 1975.

Belmont, G. and Rezeau, L.: Finite Larmor radius effects: The twofluid approach, Ann. Geophysicae, 5A, 59-70, 1987.

Chakraborty, D. and Das, K. P.: Evolution of nonlinear magnetosonic waves propagating obliquely to an external magnetic field in a collisionless plasma, J. Plasma Phys., 64, 211-226, 2000.

Chew, G. F., Goldberger, M. L., and Low, F. E.: The Boltzmann equation and the one-fluid hydromagnetic equations in the absence of particle collisions, Proc. Roy. Soc. (London), 236, 112118, 1956.

Ferrière, K. M. and André, N.: A mixed magnetohydrodynamickinetic theory of low-frequency waves and instabilities in homogeneous, gyrotropic plasmas, J. Geophys. Res., 107, SMP 7-1, 7-17, CiteID 1349, 2002.

Gazol, A., Passot, T., and Sulem, P. L.: Nonlinear dynamics of obliquely propagating Alfvén waves, J. Plasma Phys., 60, 95109, 1998.

Hasegawa, A. and Chen, L.: Kinetic processes in plasma heating by resonant mode conversion of Alfvén wave, Phys. Fluids, 19 1924-1934, 1976.

Hollweg, J. V.: Kinetic Alfvén wave revisited, J. Geophys. Res., 104, 14 811-14 819, 1999.

Janiki, M. S., Dusguspa, B., Gupta, M. R., and Som, B. K.: Solitary magnetosonic waves with Landau damping, Physica Scripta, 45, 368-372, 1992.

Kakutani, T., Ono, H., Taniuti, T., and Wei, C. C.: Reductive perturbative method in nonlinear wave propagation II, Application to hydromagnetic waves in cold plasma, J. Phys. Soc. Japan, 24, 1059-1166, 1968.

Kever, H. and Morikawa, G. K.: Korteweg-de Vries equation for nonlinear hydromagnetic waves in a warm collision-free plasma, Phys. Fluids, 12, 2090-2093, 1969.

Khanna, M. and Rajaram, R.: Evolution of nonlinear Alfvén waves propagating along the magnetic field in a collisionless plasma, J. Plasma Phys., 28, 459-468, 1982.

Kulsrud, R. M.: MHD description of plasma, in: Handbook of Plasma Physics, edited by Rosenbluth, M. N. and Sagdeev, R. Z., Vol. 1 Basic Plasma Physics edited by Galeev, A. A. and Sudan, R. N., North Holland, 115-145, 1983.

Lerche, I. and Schickeiser, R.: Linear Landau damping and wave energy dissipation in the interstellar medium, Astro. Astrophys., 366, 1008-1015, 2001a. 
Lerche, I. and Schickeiser, R.: Cosmic ray transport in anisotropic magnetohydrodynamic turbulence, Astro. Astrophys., 378, 279294, 2001b.

Li, H., Gary, S. P., and Stawicki, O.: On the dissipation of magnetic fluctuations in the solar wind, Geophys. Res. Lett., 28, 13471350, 2001.

Lithwick, Y. and Goldreich, P.: Compressible magnetohydrodynqmic turbulence in interstellar plasmas, Astrophys. J., 562, 279296, 2001.

Lysak, R. L.: Electrodynamic coupling of the magnetosphere and ionosphere, Space Sc. Rev., 52, 33-87, 1990.

Lysak, R. L. and Lotko, W.: On the kinetic dispersion relation for shear Alfvén waves, J. Gophys. Res., 101, 5085-5094, 1996.

Marchenko, V. A., Denton, R. E., and Hudson, M. K.: A magnetohydrodynamic model of kinetic Alfvén waves with finite ion gyroradius, Phys. Plasmas, 3, 3861-3863, 1996.

Minter, A. H. and Spangler, S. R.: Heating of the interstellar diffuse ionized gas via the dissipation of turbulence, Astropys. J., 485, 182-194, 1997.

Mio, K., Ogino, T., and Takeda, S.: A perturbation method and its application to obliquely propagating nonlinear Alfén waves, J. Phys. Soc. Japan, 41, 2114-2120, 1976.

Mjølhus, E. and Wyller, J.: Nonlinear Alfvén waves in a finite-betas plasma, J. Plasma Phys., 40, 299-318, 1988.

Passot, T. and Sulem, P. L.: A long-wave model for Alfvén wave trains in a collisionless plasma: I. Kinetic theory, Phys. Plasmas,
10, 3887-3905, 2003a.

Passot, T. and Sulem, P. L.: A long-wave model for Alfvén wave trains in a collisionless plasma: II, A Landau-fluid approach, Phys. Plasmas, 10, 3906-3913, 2003b.

Quataert, E., Dorland, W., and Hammett, G. W.: The magnetorotational instability in a collisionless plasma, Astrophys. J., 577, 524-533, 2002.

Rezeau, L. and Belmont, G.: Magnetic turbulence at the magnetopause, a key problem for understanding the solar wind/magnetosphere exchanges, Space, Sci. Rev., 95, 427-441, 2001.

Rogister, A.: Parallel propagation of nonlinear low-frequency waves in high- $\beta$ plasma, Phys. Fluids, 12, 2733-2739, 1971.

Sahraoui, F., Belmont, G., and Rezeau, L: Hamiltonian canonical formulation of Hall-magnetohydrodynamics: Toward an application to weak turbulence theory, Physics of Plasmas, 10, 13251337, 2003.

Snyder, P. B., Hammett, G. W., and Dorland, W.: Landau fluid models of collisionless magnetohydrodynamics, Phys. Plasmas, 4, 3974-3985, 1997.

Spangler, S. R.: The dissipation of magnetohydrodynamic turbulence responsible for interstellar scintillation and the heating of the interstellar medium, Astrophys. J., 376, 540-555, 1991.

Yajima, N.: The effect of finite ion Larmor radius on the propagation of magnetoacoustic waves, Prog. Theor. Phys., 36, 1-16, 1966. 